\title{
Au centre ou a la périphérie ? Aperçu international du rôle des bibliothèques nationales dans les réseaux de documentation At the Centre or on the Periphery: An International Perspective on the Role of National Libraries Within Library Networks ¿Al centro o a la periferia? Resumen internacional del papel de
las bibliotecas nacionales en las redes de documentación
}

\section{Graham P. Cornish}

Volume 39, numéro 2, avril-juin 1993

Les bibliothèques nationales

URI : https://id.erudit.org/iderudit/1028735ar

DOI : https://doi.org/10.7202/1028735ar

Aller au sommaire du numéro

\section{Éditeur(s)}

Association pour l'avancement des sciences et des techniques de la documentation (ASTED)

\section{ISSN}

0315-2340 (imprimé)

2291-8949 (numérique)

Découvrir la revue

\section{Citer cet article}

Cornish, G. P. (1993). Au centre ou a la périphérie ? Aperçu international du rôle des bibliothèques nationales dans les réseaux de documentation. Documentation et bibliothèques, 39(2), 53-57. https://doi.org/10.7202/1028735ar
Résumé de l'article

Après avoir défini les réseaux, l'auteur examine la participation des bibliothèques nationales à la mise en place de liens visant à créer un système national d'information. Il passe en revue des activités majeures, comme le développement de catalogues collectifs, les acquisitions, le prêt entre bibliothèques et l'édition électronique, avant de conclure à la nécessité pour les bibliothèques nationales d'être à l'avant-garde de la création des réseaux et d'éviter ainsi le confinement en périphérie.
Tous droits réservés @ $₫$ Association pour l'avancement des sciences et des techniques de la documentation (ASTED), 1993
Ce document est protégé par la loi sur le droit d'auteur. L'utilisation des services d’Érudit (y compris la reproduction) est assujettie à sa politique d'utilisation que vous pouvez consulter en ligne.

https://apropos.erudit.org/fr/usagers/politique-dutilisation/ 


\title{
Au centre ou à la périphérie? Aperçu international du rôle des bibliothèques nationales dans les réseaux de documentation
}

\author{
Graham P. Cornish \\ Chargé de programme \\ Programme de disponibilité universelle des publications de la Fédération internationale des associations de bibliothécaires
}

Après avoir défini les réseaux, l'auteur examine la participation des bibliothèques nationales à la mise en place de liens visant à créer un système national d'information. II passe en revue des activités majeures, comme le développement de catalogues collectifs, les acquisitions, le prêt entre bibliothèques et l'édition électronique, avant de conclure à la nécessité pour les bibliothèques nationales d'être à l'avant-garde de la création des réseaux et d'éviter ainsi le confinement en périphérie.

\begin{abstract}
At the Centre or on the Periphery: An International Perspective on the Role of National Libraries Within Library Networks
\end{abstract}

Following a definition of networks, the author examines the role of national libraries in the creation of links that facilitate the formation of national information systems. He summarises the principal activities such as the development of union catalogues, acquisitions, interlibraryloan and electronic publishing. He concludes that it is necessary for national libraries to be in the forefront of network creation and thus avoid being relegated to the periphery.

\section{¿Al centro o a la periferia? Resumen internacional del papel de las bibliotecas nacionales en las redes de documentación}

Después de definir las redes, el autor examina la participación de las bibliotecas nacionales en el establecimiento de lazos con el propósito de crear un sistema nacional de información. Hace una revisión de las actividades principales, como el desarrollo de los catálogos centralizados, las adquisiciones, el préstamo interbibliotecario y la edición electrónica. Por fin, insiste en la necesidad de que las bibliotecas nacionales estén en vanguardia en la créación de redes y de que eviten asi el confinamiento en periferia.
Nul doute que les «réseaux» jouissent d'une vogue professionnelle considérable. II suffit en effet qu'un article mentionne ce mot pour qu'on s'en repaisse, et ceux qui considèrent les réseaux comme une panacée mettent un zèle de prosélyte à relever les signes de la propagation du phénomène dans un nombre sans cesse croissant de pays ou dans un éventail de plus en plus large de bibliothèques.

\section{Qu'entendons-nous par réseau?}

Queveut précisément dire un réseau pour nous? Pour notre propos, un réseau est un lien qui unit plus de deux organismes à leur avantage à tous. On ne peut parler de réseau dans le cas d'un modèle purement centralisé où le centre fournit tout et les autres bibliothèques se contentent de recevoir. II serait plutôt question d'une série de liens indépendants, quoique peut-être centrés sur un établissement commun. Il est malheureux qu'on ait si étroitement associé le terme "réseau» aux systèmes automatiques. On peut encore voir de nombreux exemples de réseaux, au sens que leur donne cet article, qui sont tout à fait dépourvus de bases informatiques. Dans un certain nombre de pays en voie de développement économique, on est encore loind'une automatisation de la documentation et des activités de bibliothèque, bien qu'on y ait réussi dans certains cas à mettre en place des réseaux utiles. Cette constatation vaut aussi pour une foule de systèmes coopératifs de bibliothèque comme ceux du Royaume-Uni qui existaient bien avant que l'ordinateur ne sorte du domaine de la fiction. II est néanmoins évident que les réseaux informatisés réalisent beaucoup plus efficacement que les systèmes «manuels» certains des buts d'une mise en réseau, surtout dans la communication de données sur les sources et la rapidité de cette communication. Là où l'ordinateur n'est pas toujours la solution à un problème, le réseau représente peut-être ce remède!
Bien sûr, un réseau peut servir à communiquer toutes sortes de renseignements, des données de catalogage à l'information financière et du signalement courant aux ressources de logiciels spécialisés. Dans cet article, nous nous attacherons àl'utilisation des réseaux comme moyen de communication de renseignements sur les documents et des documents eux-mêmes. Nous ferons mention des autres usages du réseau, quelque primordiaux qu'ils soient aujourd'hui pour la bibliothèque et le service d'information, dans le contexte des perspectives professionnelles qui paraissent se dégager pour ce secteur.

Pourquoi la bibliothèque nationale devrait-elle faire partie du réseau?

Si les réseaux sont des partenariats, la bibliothèque nationale d'un pays doit être un partenaire et donc exercer une influence sur le cours des événements. 
On reconnaît généralement que, parmi les tâches d'une bibliothèque nationale, il y a le devoir d'assurer un leadership national dans les services de bibliothèque et d'information et de se trouver au coeur de ce qui se fait en bibliothéconomie et en gestion de bibliothèque ${ }^{1}$. Cette double obligation doit amener une telle bibliothèque à jouer un rôle de chef de file dans la mise en place d'un quelconque réseau. Son rôle devient primordial dans toute évolution quand on ajoute ${ }^{2}$ les tâches consistant à créer un système national d'information propre à faciliter le développement socio-économique aux niveaux individuel et national, à constituer des archives nationales, à servir de foyer à des services internationaux de fourniture de documents et à assurer une diffusion nationale des collections qu'elle possède. Pour l'essentiel, le réseau est un mécanisme de partage de ressources : documents, fiches de catalogage, données de développement des collections, service de signalement courant, bases de données et renseignements bruts. L'évolution du rôle des bibliothèques s'étendra aux ressources qu'elles veulent et doivent partager. On continue foncièrement à croire que le partage des ressources est une "bonne chose» et on note de nombreuses tentatives de lancer des projets en collaboration sur la base de cette seule perception sans jeter véritablement les fondements d'un fonctionnement efficace pour le système mis en place.

\section{Situation actuelle}

En 1990, I'UNESCO commandait une étude sur l'évolution du rôle des bibliothèques nationales dans le nouveau milieu de l'information. Le rapport produit a fait voir à propos de ces bibliothèques un grand nombre de facteurs importants, dont le moindre n'est pas leur participation à des activités essentielles à la prospérité des réseaux où elles ont une place. Nous passerons en revue plus loin quelquesunes des principales fonctions de mise en réseau.

\section{Liaison avec les catalogues d'autres} bibliothèques

Pour qu'une bibliothèque nationale s'acquitte de sa mission de diffusion nationale (par la référence, la reproduction ou le prêt) et internationale (prêt entre biblio- thèques d'originaux ou de copies) de ses collections, comme le prévoient les directives de la Fédération internationale des associations de bibliothécaires sur lesquelles il y a eu entente internationale en $1987^{3}$, il est nécessaire de relier son catalogue à ceux d'autres bibliothèques, certainement au niveau national et de plus en plus au niveau international.

Si un certain nombre de bibliothèques nationales donnent accès à leurs fichiers à l'utilisateur éloigné par un système de consultation en direct, très peu sont vraiment reliées aux catalogues d'autres bibliothèques. Si une telle liaison existe, elle peut servir à l'échange de données sur le catalogage, la procédure d'acquisition et la disponibilité d'ouvrages, ainsi qu'aux services plus familiers de catalogue collectif dont il sera question plus loin.

Souvent, il est impossible de séparer les utilisations d'une liaison, qu'il s'agisse du prêt entre bibliothèques ou d'autres fonctions. La nature et les niveaux d'une liaison varient considérablement, tout comme peuvent jouer ici le caractère et les orientations d'une «bibliothèque nationale». Dans ce qui constituait auparavant la Yougoslavie, toutes les bibliothèques nationales étaient réunies dans un réseau propre auquel appartenaient en outre beaucoup de bibliothèques universitaires. La chose existe probablement encore dans la "nouvelle» Yougoslavie, bien qu'on connaisse mal l'état actuel des bibliothèques dans certaines des ex-républiques yougoslaves. Detels liens existent également au Royaume-Uni où les trois bibliothèques nationales (British Library et bibliothèques nationales de l'Écosse et du pays de Galles) font partie de JANET (Joint Academic Network), réseau d'accès mutuel à l'information sur les fonds documentaires des membres. Dans certains petits pays, presque toutes les bibliothèques importantes sont en liaison, qu'elles aient ou non le rang de bibliothèque «nationale».

\section{Catalogues collectifs}

Un des principaux rôles d'une bibliothèque nationale devrait être de servir de foyer d'information sur les ouvrages se trouvant au pays, surtout à l'intention d'étrangers désireux d'obtenir copie de documents. Cela ne signifie pas que toutes les bibliothèques nationales jouent ce rôle adéquatement ou même qu'elles tentent de le jouer mais parfois ce désistement se justifie si la fonction peut être confiée à une autre bibliothèque nationale dans le pays. On n'a qu'à songer aux bibliothèques nationales spécialisées. Dans certains petits pays, il n'y a pas de catalogue collectif, le nombre de bibliothèques rendant inutile un tel catalogue. II se peut aussi qu'il existe d'autres catalogues collectifs que celui que tient une bibliothèque nationale. C'est ce qu'on observe, par exemple, au Royaume-Uni où on dénombre une foule de catalogues collectifs spécialisés tenus par des établissements à orientation «domaine» ou «langue», ainsi qu'un certain nombre de catalogues régionaux et de catalogues indépendants en direct. Comme nous l'avons mentionné, dans quelques pays comme l'italie, on crée et gère un catalogue collectif tout à fait en dehors des fonctions de la bibliothèque nationale (en sol italien, il y en a au moins trois qui revendiquent ce titre). II convient de noter que, dans plusieurs pays où la bibliothèque nationale ne tient pas de catalogue collectif, ni aucun autre établissement d'ailleurs, on n'est pas renseigné sur les fonds documentaires des diverses bibliothèques, d'où de graves difficultés de collaboration internationale des bibliothèques.

La structure des catalogues collectifs tenus par les bibliothèques nationales responsables varie considérablement tout comme les catalogues des collections propres de ces établissements. Dans les pays scandinaves continentaux, il existe un ensemble complexe de catalogues collectifs créés par un réseau de collaboration et embrassant un large éventail de fonds documentaires en dehors de ceux des bibliothèques nationales mises à contribution. En Suède et au Danemark, les

1. Maurice B. Line, «The Role of National Libraries: a Reassessment», Libri, vol. 30 (1980), 1-16.

2. Graham P. Cornish, Role of National Libraries in the New Information Environment, Paris, Unesco, 1991, (PG1-91/WS/4).

3. IFLA Office For International Lending, «International Lending : Principles and Guidelines for Procedure (1987)", Interlending and Document Supply, vol. 16 (1988), 28-32. Also published in IFLA Journal, vol. 14 (1988), 258-264. 
bibliothèques nationales tiennent ces catalogues, mais en Norvège et en Finlande, c'est une bibliothèque universitaire qui a repris une grande partie des tâches d'une bibliothèque nationale. En Norvège $e^{4}$ où s'implante une bibliothèque nationale avec un premier établissement dans la ville de Mo i Rana, la situation peut vite changer. En Finlande, le gros du travail de création d'un catalogue collectif se fait en collaboration. Quelle que soit la méthode retenue, les bibliothèques nationales de la Scandinavie sont toutes reliées à des catalogues collectifs électroniques que l'on peut partout interroger en direct. Mentionnons entre autres la Nordic Union Serials List ou NOSP. En Europe, on compte des catalogues collectifs consultables en direct dans nombre de pays comme les Pays-Bas, la Belgique, l'Italie, le Portugal, le Royaume-Uni, la France, la Suisse, l'Allemagne et l'Autriche. La plupart des anciens pays socialistes d'Europe orientale et centrale tiennent des catalogues collectifs en direct de périodiques étrangers. Précisons toutefois que, dans plusieurs de ces pays, la bibliothèque nationale n'établit pas les fichiers permanents ni ne maintient les bases de données, se contentant de participer à une activité qui s'exerce ailleurs.

En Amérique du Nord, il existe des modèles centralisés ou non de catalogue collectif. Ainsi, la Bibliothèque nationale du Canada tient un catalogue collectif en direct, alors qu'aux États-Unis c'est I'Online Computer Library Center (OCLC) qui remplit de facto cette fonction, ayant à toutes fins utiles le rang de catalogue national en direct. On a déjà à s'inquiéter en soi dans ce genre d'évolution que les pratiques régionales ${ }^{5}$ ne commencent à saper ce rôle national, ce qui appelle sans doute une plus grande vigilance de la part de la bibliothèque nationale.

Ailleurs dans le monde, le tableau est très inégal. En Afrique, la bibliothèque d'État de Pretoria en Afrique du Sud tient un catalogue collectif en direct pour le système de prêt entre bibliothèques de l'Afrique australe, mais ce ne sont pas toutes les autres bibliothèques nationales du réseau (Zimbabwe, Lesotho, Botswana, Namibie et Swaziland) qui ont accès à l'information pour l'instant. Très peu d'autres bibliothèques nationales africaines s'occupent actuellement de mise en réseau.
En Asie, les bibliothèques ont généralement un accès informatique restreint à des catalogues collectifs. Les grandes exceptions sont le Japon, la Malaysia, Singapour et Israël. Des catalogues collectifs verront probablement le jour dans d'autres pays avec les progrès de la mécanisation, et on en trouvera sans doute bientôt dans un certain nombre de pays arabes.

Et l'Australie et la Nouvelle-Zélande ont des catalogues collectifs complets et bien organisés reposant sur leurs réseaux bibliographiques respectifs. Dans la région, abstraction faite des îles Fidji où l'Université du Pacifique Sud joue un rôle international, il paraît peu probable qu'une autre bibliothèque nationale établisse un catalogue collectif car chaque État insulaire compte trop peu de bibliothèques.

En Amérique latine, on relève au Venezuela et au Chili6 des catalogues collectifs en direct qui se concentrent tous sur la documentation des bibliothèques universitaires. Le Mexique et le Brésil ont chacun des catalogues collectifs dans leur bibliothèque nationale, mais ils ne parrainent pas pour l'instant de catalogue d'ensemble en direct. En raison de leur petite taille, la plupart des pays des Antilles ne jugent pas d'un grand intérêt un catalogue national en direct, mais il se pourrait fort bien qu'un catalogue collectif régional voie le jour.

\section{Liens avec les réseaux}

Bien que certaines bibliothèques nationales disposent de systèmes en direct leur permettant de diffuser leurs propres catalogues et d'interroger ceux d'autres bibliothèques, ce n'est pas nécessairement pour une bibliothèque nationale une raison ou la seule raison d'appartenir à un réseau. En fait, on sait que plusieurs bibliothèques sans catalogues diffusés par réseau sont quand même en liaison avec des réseaux de bibliothèques ou avec un groupe d'autres établissements. Un bon exemple est la Thaillande. Si sa bibliothèque nationale n'a ni catalogue propre ni catalogue collectif informatisé (en réalité, elle ne possède aucun catalogue collectif à proprement parler), elle affirme être en réseau avec le Thai National Information System (THAINATIS) avec ses fonctions en sciences humaines et en sciences sociales, et aussi en liaison avec le réseau national d'échange et de prêt entre bibliothèques.

\section{Réseau et acquisition}

Un avantage de l'adhésion à un réseau est la capacité de consulter d'autres bibliothèques avant de décider d'une politique d'acquisition ou de l'achat de documents par catégorie de documentation ou par matière. C'est là un domaine où le terme "réseau» est considérablement "étiré» et se prête manifestement à des interprétations diverses. Dans maintes bibliothèques, une telle adhésion n'est pas un critère dans l'établissement des politiques d'acquisition, bien qu'il y ait le cas échéant consultation d'autres organismes. Cela vaut particulièrement pour les supports d'information. Beaucoup de bibliothèques nationales n'achètent ni films, ni vidéos, ni enregistrements sonores qu'elles voient comme une responsabilité des archives nationales du son ou du film. Dans des pays comptant un certain nombre de centres nationaux spécialisés comme l'Allemagne, on s'entend officiellement sur les acquisitions àl'échelle nationale. Au Royaume-Uni, la British Library n'acquiert pas de documents en gallois ni en gaélique, cettetâche revenant aux bibliothèques nationales respectives du pays de Galles et de l'Écosse. Toutefois, les bibliothèques nationales ne sont pas appelées à recourir aux catalogues collectifs ni aux réseaux pour vérifier les politiques d'acquisition des autres grandes bibliothèques d'un pays ou d'une région. La raison en est peut-être que la bibliothèque nationale se doit d'être complète, du moins en ce qui concerne les documents émanant du territoire national. On voit donc mieux les autres bibliothèques

4. Libena Vokac, «The National Library of the North: Norway's New National Library Branch at Mo i Rana and its Future Role in Lending», dans Alison Gallico, Interlending and Document Supply: Proceedings of the Second International Conference held in London, November 1990 , Boston Spa (UK), British Library and IFLA Office for International Lending, 1991, p. 65-67.

5. M. Jackson, «Library to Library», WilsonLibrary Bulletin, vol. 63 no. 8 (1989), 88-89.

6. Patricio Del Sol et al., "Acquisition and Cooperative Use of Serials», Interlending and Document Supply, vol. 19 (1991) 131-137. 
vérifier auprès de la bibliothèque nationale avant d'acheter que le contraire. II devrait sûrement en être ainsi pour les publications nationales dont le dépôt obligatoire devrait permettre de constituer des archives nationales complètes, mais cela vaut beaucoup moins pour la documentation étrangère. Ces derniers documents étant de plus en plus chers, surtout dans les pays connaissant des problèmes de devises, la nécessité d'une consultation, de préférence à l'aide de systèmes en direct, deviendra encore plus grande si bien que la gestion du développement des collections acquerra un caractère global laissant la bibliothèque nationale se concentrer sur ses propres obligations et donnant la liberté aux autres bibliothèques d'être plus sélectives dans leurs programmes d'acquisition.

\section{Réseau et prêt entre bibliothèques}

Peut-être l'application des réseaux que l'on apprécie et comprend le plus directement est-elle celle du prêt entre bibliothèques et de la fourniture de documents. Les services de prêt et de fourniture des bibliothèques nationales sont fort variables. Les bibliothèques qui s'adonnent au prêt entre bibliothèques ne font pas toujours partie d'un réseau d'où des difficultés d'exploitation de leurs fonds d'information et de leurs installations. Le meilleur guide des centres de prêt international ${ }^{7}$ indique que beaucoup de bibliothèques nationales, surtout dans des pays où les systèmes d'information sont en développement, ne prennent nulle part au prêt international entre bibliothèques ni même à l'exploitation des systèmes nationaux de prêt. En Europe, les pays scandinaves, l'Allemagne, le Royaume-Uni, les PaysBas et l'Autriche ont tous un rôle de chef de file dans les systèmes de prêt entre bibliothèques, mais plus on gagne l'Europe méridionale, plus ce rôle s'atténue. On relève des différences semblables entre l'Amérique du Nord et l'Amérique du Sud. Parmi les autres pays où la bibliothèque nationale est un important intervenant, mentionnons l'Australie, la NouvelleZélande, la Malaysia et l'Afrique du Sud. Dans bien des pays ou des régions, on a toujours la notion que la bibliothèque nationale est autant un dépôt d'archives qu'un service dynamique de diffusion directe de documents.
Il convient de noter que, en adhérant à un service comme celui de I'OCLC, des bibliothèques nationales donnent à leur documentation une diffusion aussi bien internationale que nationale. Le projet commun du Royaume-Uni, des Pays-Bas et de la France permettra en outre une liaison internationale pour les demandes de prêts entre bibliothèques.

\section{Liaisons prévues}

Si la mise en réseau doit relever d'une bibliothèque nationale, on peut espérer que les bibliothèques nationales qui ne se sont pas jointes à un réseau et qui n'en ont pas établi non plus feront bientôt quelque chose en ce sens. Autant que nous le sachions, seules quatre bibliothèques nationales, à savoir celles du Brésil, de la Bulgarie, de l'Iran et du Mexique, ont déclaré n'avoir aucun projet immédiat d'extension ni de création de réseau. En revanche, beaucoup d'autres bibliothèques nationales ont fait mention de plans d'avenir dans ce domaine. Leur répartition géographique est intéressante. Bien qu'on voie l'Amérique du Nord et l'Europe comme le berceau de l'informatisation et de la mise en réseau des bibliothèques, on compte plus de bibliothèques en Europe prévoyant des travaux de mise en réseau qu'en Afrique ou en Asie.

$\begin{array}{lr}\text { Afrique } & 9 \\ \text { Asie } & 11 \\ \text { Europe } & 12 \\ \text { Amérique latine } & 1\end{array}$

Comme certains des pays évoquant des plans en ce sens sont très petits et ont peu de bibliothèques, la liaison prévue sera peut-être plus régionale que nationale.

Le tableau général qui se dégage est celui d'une évolution rapide dans les pays où des réseaux sont en place et d'un intérêt actif dans les pays qui en sont encore au stade de la planification. II est encourageant de constater les plans de développement dans des pays qui n'ont pas passé le cap du «catalogue manuel». II paraît probable que beaucoup de pays feront le saut des services primitifs de bibliothèque à la haute technologie, exemple qu'ont donné les États insulaires du Pacifique Sud dans bien des secteurs, et notamment dans ceux de la communication par satellite et de la télécopie.

\section{Perspectives d'avenir}

\section{Leadership et participation}

Le leadership national est une question plus de qualité et de motivation que de technologie! Toutefois, si une bibliothèque nationale prend l'initiative de progresser technologiquement, il est probable qu'elle se trouvera établie dans ce rôle pour longtemps. Dans un pays, le nombre d'établissements s'occupant de technologie de bibliothèque est restreint et la bibliothèque nationale a l'occasion de frayer la voie dans ce domaine. Si elle ne sait pas saisir cette occasion, elle régresse en matière technologique et abandonne son leadership à d'autres subissant des effets néfastes à long terme sur sa réputation dans le monde des bibliothèques et des services d'information.

\section{Le défi du succès}

Une tâche de plus en plus importante pour une bibliothèque nationale sera d'assurer un service d'information national pour faciliter l'évolution socio-économique au niveau tant national qu'individuel. L'utilisation de bases en direct, le recours à des fonds complets de référence, peutêtre stockés sur disque compact, et le fichage électronique de ses propres collections, voilà autant de moyens qui s'offrent à elle de procurer un large éventail de ressources d'information, ce qui est tout à fait impossible à l'établissement qui ne sait se servir du progrès technique. L'emploi de telles installations peut assurer à un pays une riche gamme de sources d'information et de documentation à exploiter pour le développement tant national qu'individuel. Le défi que doivent relever les bibliothèques nationales est celui du succès. Si l'utilisation des installations s'accroît, on demandera inévitablement plus de services. De même, les services susciteront souvent une demande de documents n'appartenant pas à la bibliothéque nationale ( $\mathrm{ni}$ à aucune autre bibliothèque du pays). La stimulation des services de fourniture de documents

7. Margaret M. Barwick, A Guide to Centres of International Lending and Copying, $4^{\text {th }}$ ed., Boston Spa (UK), IFLA Office for International Lending, 1990. 
par une utilisation accrue des supports électroniques est un important aspect à prendre en considération quand on introduit de tels services. II est aussi loisible à une bibliothèque nationale de recourir au télétexte pour une vaste gamme de services d'information, souvent avec des retombées indirectes comme une meilleure sensibilisation de la population au rôle et aux services d'un établissement semblable.

\section{Défi de l'édition électronique}

La production électronique de documents offre aux bibliothèques nationales un important défi susceptible de renforcer ou de détruire leur rôle de chef de file dans leur pays. Souvent, les documents électroniques ne font pas l'objet d'exigences légales de dépôt, bien que le législateur ait adopté des mesures en ce sens dans des pays comme la Norvège ${ }^{8}$. Si les documents électroniques ne sont pas déposés, une bibliothèque nationale deviendra de plus en plus un musée du document imprimé pendant que d'autres auront les moyens de se doter de systèmes d'information complexes comportant la documentation électronique. Disons par ailleurs que l'acquisition de documents électroniques pose eile aussi des problèmes en matière de propriété, d'accès, de manipulation ou même de possibilités de réédition et de reconditionnement. D'un point de vue technique, l'édition électronique est un des meilleurs modes de diffusion puisqu'on peut théoriquement avoir accès de partout à un texte en archivage central si on dispose du matériel et des moyens de télécommunication nécessaires. Précisons toutefois que l'entreposage de textes électroniques à la bibliothèque nationale devrait sans doute se faire dans des conditions très strictes et notamment avec une interdiction de mise en réseau, voire de communication à la demande. Avec de la bonne volonté et de la compréhension de part et d'autre, on peut résoudre les nombreux problèmes juridiques de l'édition électronique, mais ce n'est pas encore chose faite et la démarche sera entravée par l'absence dans la loi de dispositions claires sur le droit d'auteur et le dépót légal. II faut espérer que les nouveaux travaux de réforme de la loi canadienne sur le droit d'auteur permettront de s'attaquer à ces questions et de clarifier le rôle non seulement des bibliothèques nationales, mais aussi de tous les bibliothécaires s'occupant de documents électroniques.

\section{Défi du nouveau milieu de l'information}

L'information est une industrie majeure quelle que soit la définition qu'on en donne. Le désir de l'information (sans parler de sa nécessité) est une constante caractéristique des orientations culturelles aujourd'hui, plus particulièrement dans le monde industrialisé. L'information se présente sous diverses formes : journaux, revues, livres, émissions de radio-télévision, télétexte et systèmes en direct. La demande est là et les bibliothèques nationales doivent s'assurer qu'elles contribuent à y répondre comme elles le devraient, quelle que soit la forme qu'elle prenne. Dans les hauts lieux de l'industrie de la communication de l'information, on n'a pas simplement rebaptisé les bibliothécaires «scientifiques de l'information», mais on les a bombardés «scientifiques de la connaissance». Un tel spécialiste de la connaissance est appelé non seulement à fournir de l'information, mais aussi à l'interpréter pour le client. Cette orientation particulière amène les intéressés à recevoir des demandes de données appropriées sous une forme adaptée sur une question ou un aspect d'une question. Le produit fourni peut être un jeu de statistiques, des compilations de données, des textes juridiques, de l'information sur les sociétés, des prévisions économiques ou d'autres éléments de documentation. II peut s'agir d'une combinaison de documents, de photos, d'enregistrements sonores, d'images mobiles et d'éléments plus éphémères. Un scientifique de la connaissance sera tenu de puiser ces renseignements à toutes les sources utiles et, pour sa part, le client s'intéressera peu à l'origine du document qu'il reçoit si celui-ci répond à ses besoins de l'heure. On se trouve à un extrême du phénomène de la communication de l'information, mais cet aspect demeure révélateur d'un mouvement qui se dessine de plus en plus dans l'industrie de l'information à tous les niveaux. On voit dans les documents des "véhicules» d'information au sens le plus large, et on prête une acception tout aussi générale au terme «information». L'utilisation d'audiocassettes dans les moyens de transport en commun (comme succédané à la lecture) ou dans la voiture est un net indice de cette attitude (espérons que l'audiocassette n'est pas là pour remplacer la lecture, mais pour la compléter).

La seule façon de relever ces défis sera de créer des réseaux. Aucune bibliothèque ne peut réunir tous les documents nitoutes les compétences, aucunene peut prévoir les besoins de tous ses clients (utilisateurs), et encore moins un grand dépost à vocation générale comme une bibliothèque nationale. Le défi sera d'édifier des collections d'intérêt et de donner accès à des établissements plus compétents aux services complémentaires. Une bibliothèque nationale ne peut prétendre tout faire ni essayer d'y parvenir. Elle n'en devrait pas moins être un centre d'excellence sur le plan de la documentation, de l'information et de la connaissance en devenant le foyer d'information sur tous les documents disponibles pour la continuité de la quête du savoir.

La bibliothèque nationale a la possibilité de jouer plus qu'un rôle symbolique comme pivot du développement des bibliothèques. En se tenant à l'avant-garde de la mise en place de réseaux et de systèmes d'information en direct, elle peut littéralement se situer au centre de tout ce qui se fait dans ce domaine. En relevant le défi, elle peut se donner un rôle qui résistera à tous les assauts, sinon elle laissera d'autres, rappelons-le, usurper ce rôle en permanence. Elle sera confinée à la périphérie du mouvement au lieu d'en occuper le coeur.

8. Kristen Engelstad, «Ny lov am avleveringsplikt for allment tilgjengelihe dokumenten, Synopsis, vol. 21 (1990), 157-161. 